\title{
Comparison of robotic and laparoscopic partial nephrectomy for small renal tumours
}

\author{
Abdulmuttalip Simsek, Abdullah Hizir Yavuzsan, Yunus Colakoglu, Arda Atar, \\ Selcuk Sahin, Volkan Tugcu \\ Bakirkoy Sadi Konuk Research and Training Hospital, Department of Urology, Istanbul, Turkey.
}

\begin{abstract}
Summary Objective: To evaluate a single surgeon oncological and functional outcomes of laparoscopic partial nephrectomy (LPN) compared to robotic partial nephrectomy (RPN) for pTla renal tumours.

Materials and methods: Between 2006 and 2016, a retrospective review of 42 patients who underwent LPN $(n=20)$ or RPN $(n=22)$ by same surgeon was performed. Patients were matched for gender, age, body mass index (BMI), American Society of Anaesthesiologists (ASA) score, tumour side,

RENAL and PADUA scores, peri-operative and post-operative outcomes.

Results: There was no significant differences between the two groups with respect to patient gender, age, BMI, ASA score, tumours side, RENAL and PADUA scores. Mean operative time for RPN was 176 vs. 227 minutes for LPN ( $p=0.001)$. Warm ischemia time was similar in both groups $(p=0.58)$. Estimated blood loss (EBL) was higher in the LPN. There was no significant difference with preoperative and postoperative creatinine and percent change in eGFR levels. Only one case in LPN had positive surgical margin.

Conclusions: RPN is a developing procedure, and technically feasible and safe for small-size renal tumours. Moreover RPN is a comparable and alternative operation to LPN, providing equivalent oncological and functional outcomes, as well as saving more healthy marginal tissue and easier and faster suturing.
\end{abstract}

KEY WORDS: Partial nephrectomy; Robotic; Laparoscopic; Small renal tumour.

Submitted 18 March 2017; Accepted 23 April 2017

\section{INTRODUCTION}

Renal cell carcinoma (RCC) is one of the common cancer and represents $2-3 \%$ of all cancers (1), with the highest incidence in western countries. There is a male predominance with a peak incidence between 60-70 years. Aetiology factors include smoking, obesity, hypertension, acetaminophen, and viral hepatitis (2). Using new diagnostic tools the tumour size has decreased throughout the years and open partial nephrectomy has become an efficacious alternative to radical nephrectomy for small RCC. Partial nephrectomy, in patients who have a solitary kidney, bilateral RCC, genetic disease with RCC, and small sized tumour with a normal function contra lateral kidney, has an important advantage for preserva- tion of renal function that can have long term association with patients overall health (3).

The laparoscopic approach has been performed since 1993, showing to provide functional and oncologic outcomes equivalent to those of open surgery and affording the patients a shorter hospital stay and more rapid recovery time $(4,5)$. However, the laparoscopic technique remains difficult for the average urologist, because of the technical challenge of intracorporeal suturing. Therefore, complex laparoscopic partial nephrectomy is only limited to experienced surgeons at high volume centres (6). The introduction of robotic technology allows for complex procedures to be performed more easily by most of surgeons without experience than the conventional laparoscopic approach. This technology has revolutionized the surgical management of prostate cancer, then has been successfully utilized for pyeloplasty, radical and partial nephrectomy (7-9). Robotic surgery has some technical advantages such as magnified visualization, 3Dimensional visualization, fully articulating instruments under precise control, absence of the fulcrum effect, and elimination of tremors. These details decrease the technical difficulty associated with critical portion of partial nephrectomy including tumour dissection and pelvicalyceal renal reconstructions.

In the present study we aimed to retrospectively compare a single surgeon experience with laparoscopic and robotic partial nephrectomy for suspected RCC.

We evaluated intraoperative and postoperative parameters to compare the two methods.

\section{MATERIALS AND Methods}

Clinical data of patients who underwent robotic assisted laparoscopic partial nephrectomy (RPN) or laparoscopic partial nephrectomy (LPN) by a single surgeon between 2006 and 2016 at Bakirkoy Dr. Sadi Konuk Training and Research Hospital were obtained from medical record system. We reviewed data of 60 patients who underwent robotic or laparoscopic partial nephrectomy. Inclusion criteria were a single renal mass $\leq 4 \mathrm{~cm}$ and follow up for $\geq 3$ months after surgery. Of the 60 patients, 17 cases were excluded from the study because of $>4 \mathrm{~cm}$ tumour, zero ischemia tumour excision, conversion to open surgery, metastatic disease, a solitary kidney, multiple tumours, or 
loss to follow up. Retrospective analysis was performed for 42 patients, of which 22 (52.3\%) and 20 (47.7\%) underwent RPN and LPN, respectively.

We reviewed medical data's for patient age, sex, body mass index, consumption of tobacco, previous surgical history, American Society of Anaesthesia physical status score (ASA score), Charlson Comorbidity Index, tumor laterality, preoperative and postoperative $3^{\text {th }}$ month serum creatinine level. RENAL nephrometry score (10) and PADUA score (11) were calculated by preoperative contrast-enhanced computed tomography. Operation time, warm ischemic time (WIT) and estimated blood loss (EBL) were evaluated. Estimated glomerular filtration rate (eGFR) was calculated to evaluate renal function preoperatively and at $3^{\text {th }}$ month after the surgery (MDRD-GFR) (12). Perioperative and postoperative complications were assessed using the Clavien-Dindo Classification (13).

\section{Surgical technique}

For all cases, the patient is placed in flank position. For robotic procedures, a 4-arm approach was used, and the da Vinci S HD and the da Vinci Xi systems (Intuitive Surgical, Sunnyvale, CA) were used. For the da Vinci $S$ HD system we used the same technique that has been previously described (14). After starting to use the da Vinci Xi system we have changed the port placement because of lesser clashing of the robotic arms. All four robotic trocars were inserted through the midaxillary line. Assistant port was located medially near the umbilicus. For laparoscopic procedures traditional laparoscopic port configuration for renal surgery was used.

After starting the operation, the renal hilum was dissected and the tumour was identified. Renal arteries were clamped with laparoscopic bulldog clamps. In the robotic cases the assistant surgeon replaced the bulldog clamps. Then tumour was excised by cold scissors.

The tumour bed was continuously sutured by 3-0 selfretaining absorbable barbed suture. Parenchymal defect was sutured by 1-0 vicryl suture with Hemolock clips attached (15). A silicone drain was replaced and the operation was finished.

Statistical analysis was performed using SPSS software programme. Date are expressed as the mean \pm standard deviation or as a percentage of baseline, chi-square and independent- sample t-test were used and a $\mathrm{p}$ value of $<0.05$ was considered to indicate statistically significance.

\section{RESULTS}

A total of 42 patients (20 robotic, 22 laparoscopic partial nephrectomy) participated in the study. The mean follow-up of the robotic and laparoscopic groups were 35.4 \pm 7.3 and $49.1 \pm 12.6$ months, respectively. The patient's demographic characteristics are summarized in Table 1. There were not any statistically significant differences in the baseline characteristics among the groups regarding age, body mass index (BMI), CCI, ASA class, tumour size, RENAL, and PADUA scores.

Table 2 presents the perioperative features and change in serum creatinine levels and eGFR of the two groups. Patients in the robotic group had shorter operative time in comparison to laparoscopic groups (176 vs. 227.5,
Table 1.

Pre-operative patients data.

\begin{tabular}{|lccc|}
\hline & LPN & RPN & P value \\
\hline Patients $(\mathrm{n})$ & 20 & 22 & \\
Male & 15 & 12 & 0.20 \\
Female & 5 & 10 & \\
\hline Mean age $(\mathrm{y})$ & $50.2 \pm 11.3$ & $54.8 \pm 9.6$ & 0.16 \\
\hline Mean BMI $\left(\mathrm{kg} / \mathrm{m}^{2}\right)$ & $27.7 \pm 3.5$ & $27.3 \pm 4.9$ & 0.76 \\
\hline $\mathbf{C C l}$ & $2.3 \pm 0.8$ & $2 \pm 0.4$ & 0.12 \\
\hline ASA score & $1.9 \pm 0.4$ & $1.9 \pm 0.7$ & 0.99 \\
\hline Smokers $(\mathrm{n})$ & 14 & 16 & \\
\hline Surgical history $(\mathrm{n})$ & 8 & 6 & \\
\hline Side $(\mathrm{n})$ & \multicolumn{4}{|c}{} \\
$\begin{array}{l}\text { Left } \\
\text { Right }\end{array}$ & 14 & 13 & 0.53 \\
\hline Mean RENAL score & $5.2 \pm 1.4$ & $4.6 \pm 1.2$ & 0.14 \\
\hline Mean PADUA score & $6.4 \pm 2.1$ & $6.2 \pm 1.6$ & 0.72 \\
\hline BMI: Body Mass Index; CCl: Charlson Comorbidity Index; & \\
ASA score: American Society of Anesthesia physical status score. & \\
\hline
\end{tabular}

Table 2.

Peri-operative and post-operative outcomes.

\begin{tabular}{|c|c|c|c|}
\hline & RPN & LPN & $P$ value \\
\hline Operation time, min & $176 \pm 23.6$ & $227.5 \pm 56.3$ & 0.001 \\
\hline WIT, min & $16.2 \pm 6.7$ & $17.6 \pm 9.4$ & 0.58 \\
\hline EBL, CC & $182.5 \pm 50.4$ & $218.8 \pm 60.7$ & 0.04 \\
\hline Hospital stay (days) & $6.1 \pm 2.4$ & $4.4 \pm 1.9$ & 0.01 \\
\hline Positive surgical margin & 0 & 1 & \\
\hline Transfusion & 0 & 1 & \\
\hline \multicolumn{4}{|l|}{ Serum creatinine, $\mathrm{mg} / \mathrm{dl}$} \\
\hline Preoperative & $0.81 \pm 0.12$ & $0.85 \pm 0.19$ & 0.42 \\
\hline Postoperative $3^{\text {th }}$ month & $0.95 \pm 0.35$ & $0.97 \pm 0.24$ & 0.82 \\
\hline \multicolumn{4}{|l|}{ eGFR } \\
\hline Preoperative & $104.65 \pm 28.6$ & $89.2 \pm 24.5$ & 0.06 \\
\hline Postoperative $3^{\text {th }}$ month & $85 \pm 19.3$ & $75.2 \pm 14.5$ & 0.06 \\
\hline
\end{tabular}

$\mathrm{p}<0.01)$. Subjects in the LPN group had greater EBL (182.5 vs. 218.8, $\mathrm{p}<0.05)$ and shorter hospital stay (4.4 vs. 6.1, p < 0.05) compared to the robotic approach.

We did not detect any statistical significant differences between the groups including postoperative $\mathrm{Hb}$, transfusion rates, days of drain preservation, eGFR changes, percent of patients with Clavien-Dindo complications and mortality rates (Figure 1).

There was only one major complication in LPN group. This patient had urinary leakage at the first day of the operation.

\section{Figure 1.}

Postoperative complication $C D C$ grade $\mathrm{I}-\mathrm{Il}$.

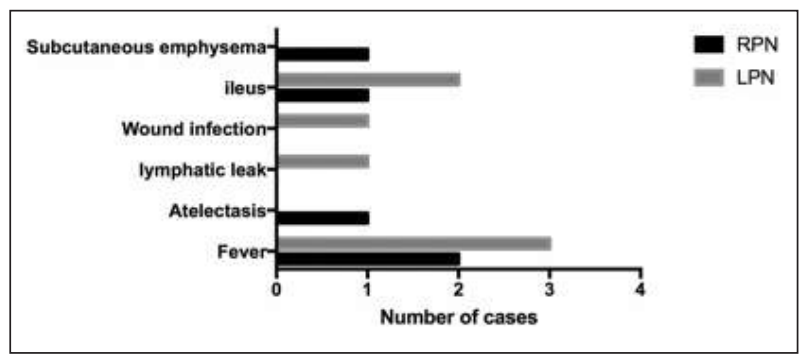


At first the patient followed conservatively then ureteral stent was replaced because of the continuation of urinary leakage.

\section{Discussion}

The number of renal cell carcinoma that are diagnosed each year is increasing owing to development and increased use of new imaging modalities. At the same time the number of small RCC cases has also risen. Following recent development of surgical instruments and techniques, partial nephrectomy has become the method of choice for the management of $\mathrm{Tl}$ renal masses. Nephron sparing surgery has many advantages for preservation of renal function and overall survival in cases of Tla cases (16). Laparoscopic partial nephrectomy (LPN) offers a shorter convalescence, reduced need for analgesia, and comparable outcomes to open partial nephrectomy in the expert surgeons (17). On the other hand, LPN can be particularly challenging for complex tumours, such as endophytic, and hilar masses. For these cases tumour resection and repairing the defect under the time constraints of warm ischemia is difficult (7). RPN is a viable alternative of more technical challenging LPN, since the da Vinci robot system has advantages such as the seven degree of freedom, high definition imaging, easily movement, stable motion for tremor, resection and suturing can be performed easier and faster (18). Disadvantages of robotic surgery are lack of tactile feedback, high cost, and time consumed for the setting up the robot (19). Moreover, the surgeon is unscrubbed in the operation console and thus could not proceed to the operating table immediately in an emergent situation (20).

LPN or RPN has the following three main goals: oncologic control, preservation of renal function and a low morbidity. In 2012, Buffi et al. proposed a simple classification system to identify patients with the optimal outcomes after PN procedures. They combined the three main goals of PN into the margin, ischemia and complication system. The background of the this system was as follows: (1) the margin is a surrogate for determining whether the primary tumour is completely removed; (2) ischemia (in particular, the warm ischemia time) is the surgical variable that influences the postoperative renal function, and (3) the modified Clavien-Dindo classification is a measure of the safety profile of PN. According to this system, the goal of PN is achieved when (1) the surgical margins are negative, (2) the WIT is $<20 \mathrm{~min}$ and (3) no major complications (grade 3-4 according to Clavien classification) are observed (21).

Warm ischemia time in the partial nephrectomy is one of the main concerns. Clamping of the renal vascular supply reduces bleeding from the resection margin, however can cause permanent ischemia injury in the renal parenchyma. For this reason warm ischemia time should be less than 30 minutes (7). Large intraparenchymal or hilar tumour usually needs more time, and can be resected efficiently and repair faster with robotics. In the series of Haber et al. study comparing LPN and RPN cases, they concluded that there were no significant differences with respect to warm ischemic time (18.2 minutes vs 20.3 minutes, respectively) (22). In our study RPN was asso- ciated with shorter operation time and warm ischemic time, but WIT was not statistically significant.

Oncologic outcome with negative surgical margin is the primary focus of partial nephrectomy. However, impact of positive margin on the oncological outcome of patients with RCC remains controversial (23). Our single institution, single surgeon series demonstrated that lower positive margin rate were seen in the RPN group. We found no difference in the incidence of PSM when comparing the clampless procedures, and no pre- or perioperative parameters predicted the PSM in both groups.

The more widespeared use of grading system for reporting complications has developed by Dindo et al. (13) to facilitate standardization. The safety of clampless PN emerged from analysis of the postoperative complications. The overall complication rates were similar for the clampless LPN and RPN groups in terms of the incidence of serious, in particular grade III-IV complications according to Clavien-Dindo classification have not seen in LPN or RPN. The PADUA and RENAL scores are important predictor of the overall complication. This study confirms that the anatomical and topographical characteristics of the tumour expressed by the PADUA or RENAL score did not affect the outcomes of RCC less than $4 \mathrm{~cm}$.

Retrospective study design was the main limitation of this study. Another limitation is that all results were based on same surgeon's experiences. A third limitation is that the study group is too small. To better elucidate comparison of LPN and RPN, a sample of large size studied in a prospectively randomized controlled design with long term follow up data will be necessary to determinate whether LPN or RPN is safe, reproducible and effective according to renal function and oncological outcomes. In conclusion, robotic surgery in urology is increasingly replacing the conventional urological techniques.

Our results demonstrated that favourable results of RPN compared to LPN in the perioperative outcomes of estimated blood loss, shorter warm ischemia time, and less positive margin rate. Besides higher costs, RPN is safe, feasible, and associated low morbidity for small-sized renal tumours.

\section{REFERENCES}

1. Lindblad P Epidemiology of renal cell carcinoma. Scand J Surg. 2004; 93:88-96.

2. Sun M, Becker A, Tian Z, et al. Management of localized kidney cancer: calculating cancer-specific mortality and competing risks of death for surgery and nonsurgical management. Eur Urol. 2014; 65:235-41.

3. Snow DC, Bhayani SB. Rapid communication: chronic renal insufficiency after laparoscopic partial nephrectomy and radical nephrectomy for pathologic tla lesions. J Endourol. 2008; 22:337-41.

4. McDougall EM, Elbahnasy AM, Clayman RV. Laparoscopic wedge resection and partial nephrectomy--the Washington University experience and review of the literature. JSLS. 1998; 2:15-23.

5. Gill IS, Kavoussi LR, Lane BR, et al. Comparison of 1,800 laparoscopic and open partial nephrectomies for single renal tumors. $J$ Urol. 2007; 178:41-6. 
6. Desai MM, Gill IS, Kaouk JH, et al. Laparoscopic partial nephrectomy with suture repair of the pelvicaliceal system. Urology. 2003; 61:99-104.

7. Gettman MT, Blute ML, Chow GK, et al. Robotic-assisted laparoscopic partial nephrectomy: technique and initial clinical experience with DaVinci robotic system. Urology. 2004; 64:914-8.

8. Peschel R, Neururer R, Bartsch G, Gettman MT. Robotic pyeloplasty: technique and results. Urologic Clin North Am. 2004; 31:737-41.

9. Guillonneau B, Jayet C, Tewari A, Vallancien G. Robot assisted laparoscopic nephrectomy. J Urol 2001; 166:200-1.

10. Kutikov A, Uzzo RG. The R.E.N.A.L. nephrometry score: a comprehensive standardized system for quantitating renal tumor size, location and depth. J Urol. 2009; 182:844-53.

11. Ficarra V, Novara G, Secco S, et al. Preoperative aspects and dimensions used for an anatomical (PADUA) classification of renal tumours in patients who are candidates for nephron-sparing surgery. Eur Urol. 2009; 56:786-93.

12. K/DOQI clinical practice guidelines for chronic kidney disease: evaluation, classification, and stratification. Am J Kidney Dis. 2002; 39:S1-266.

13. Dindo D, Demartines N, Clavien PA. Classification of surgical complications: a new proposal with evaluation in a cohort of 6336 patients and results of a survey. Ann Surg. 2004; 240:205-13.

14. SB1 B. da Vinci robotic partial nephrectomy for renal cell carcinoma: an atlas of the four-arm technique. J Robot Surg. 2008; 1:279-85.

15. Benway BM WA, Cabello JM, Bhayani SB. Robotic partial nephrectomy with sliding-clip renorrhaphy: technique and outcomes. Eur Urol. 2009; 55:592-9.

16. Zini L, Perrotte $P$, Capitanio $U$, et al. Radical versus partial nephrectomy: effect on overall and noncancer mortality. Cancer. 2009; 115:1465-71.

17. Riggs SB, Larochelle JC, Belldegrun AS. Partial nephrectomy: a contemporary review regarding outcomes and different techniques. Cancer J. 2008; 14:302-7.

18. Wang AJ, Bhayani SB. Robotic partial nephrectomy versus laparoscopic partial nephrectomy for renal cell carcinoma: singlesurgeon analysis of > 100 consecutive procedures. Urology. 2009; 73:306-10.

19. Phillips CK, Taneja SS, Stifelman MD. Robot-assisted laparoscopic partial nephrectomy: the NYU technique. J Endourol. 2005; 19:441-5.

20. Stifelman MD, Caruso RP, Nieder AM, Taneja SS. Robot-assisted laparoscopic partial nephrectomy. JSLS 2005; 9:83-6.

21. Buffi N, Lista G, Larcher A, et al. Margin, ischemia, and complications (MIC) score in partial nephrectomy: a new system for evaluating achievement of optimal outcomes in nephron-sparing surgery. Eur Urol. 2012; 62:617-8.

22. Haber GP, White WM, Crouzet S, et al. Robotic versus laparoscopic partial nephrectomy: single-surgeon matched cohort study of 150 patients. Urology. 2010; 76:754-8.

23. Khalifeh A KJ, Bhayani S, Rogers C, et al. Positive surgical margins in robot-assisted partial nephrectomy: a multi-institutional analysis of oncologic outcomes (leave no tumor behind). J Urol. 2013; 190:1674-9.

\section{Correspondence}

Abdulmuttalip Simsek, MD (Corresponding Author) simsek76@yahoo.com

Abdullah Hizir Yavuzsan, MD

hiziryavuzsan@yahoo.com

Yunus Colakoglu, MD

yunuscolakoglu@gmail.com

Arda Atar, MD

ardatar@yahoo.com

Selcuk Sahin, MD

selcuksahin@yahoo.com

Volkan Tugcu, MD

volkantugcu@yahoo.com

Department of Urology, Bakirkoy Sadi Konuk Research

and Training Hospital

Zuhuratbaba, Tevfik Saglam Cad. NO:11

Bakirkoy, Istanbul, Turkey 